\title{
Rapid identification of mutations caused by fast neutron bombardment in Medicago truncatula
}

Huan Du ${ }^{1,2+}$, Zhicheng Jiao ${ }^{1,2+}$, Junjie Liu ${ }^{1,2}$, Wei Huang ${ }^{3}$ and Liangfa Ge $e^{1,2,4^{*}}$ (D)

\begin{abstract}
Background: Fast neutron bombardment (FNB) is a very effective approach for mutagenesis and has been widely used in generating mutant libraries in many plant species. The main type of mutations of FNB mutants are deletions of DNA fragments ranging from few base pairs to several hundred kilobases, thus usually leading to the null mutation of genes. Despite its efficiency in mutagenesis, identification of the mutation sites is still challenging in many species. The traditional strategy of positional cloning is very effective in identifying the mutation but time-consuming. With the availability of genome sequences, the array-based comparative genomic hybridization (CGH) method has been developed to detect the mutation sites by comparing the signal intensities of probes between wild-type and mutant plants. Though CGH method is effective in detecting copy number variations (CNVs), the resolution and coverage of CGH probes are not adequate to identify mutations other than CNVs.

Results: We report a new strategy and pipeline to sensitively identify the mutation sites of FNB mutants by combining deep-coverage whole-genome sequencing (WGS), polymorphism calling, and customized filtering in Medicago truncatula. Initially, we performed a bulked sequencing for a FNB white nodule (wn) mutant and its wild-type like plants derived from a backcross population. Following polymorphism calling and filtering, validation by manual check and Sanger sequencing, we identified that SymCRK is the causative gene of white nodule mutant. We also sequenced an individual FNB mutant yellow leaves 1 (y/1) and wild-type plant. We identified that ETHYLENE-DEPENDENT GRAVITROPISM-DEFICIENT AND YELLOW-GREEN 1 (EGY1) is the candidate gene for M. truncatula yl1 mutant.
\end{abstract}

Conclusion: Our results demonstrated that the method reported here is rather robust in identifying the mutation sites for FNB mutants.

Keywords: Fast neutron bombardment, Medicago truncatula, Next-generation sequencing, Mutation

\section{Introduction}

Plant mutant collections are very useful genetic resources and widely used in forward and reverse genetic studies. Mutants can be induced by different mutagens, such as

\footnotetext{
*Correspondence: Ige@scau.edu.cn

${ }^{\dagger}$ Huan Du and Zhicheng Jiao authors contributed equally to the work

${ }^{1}$ Guangdong Key Laboratory for Innovative Development and Utilization of Forest Plant Germplasm, College of Forestry and Landscape

Architecture, South China Agricultural University, Guangzhou 510642, Guangdong, China

Full list of author information is available at the end of the article
}

chemical agents or biological factors. Ethyl methane sulfonate (EMS) and Transfer DNA (T-DNA) are two popular mutagens that typically induce point nucleotide substitution and T-DNA insertional mutation, respectively [1-4]. Although EMS mutation is relatively easy to be generated, it always induces numerous background mutations and thus requires substantial efforts to identify causative mutation sites. By contrast, T-DNA insertion mutations typically harbor fewer background mutations and the mutation sites could be identified by the flanking sequences theoretically. However, T-DNA mutation

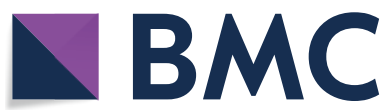

(c) The Author(s) 2021. This article is licensed under a Creative Commons Attribution 4.0 International License, which permits use, sharing, adaptation, distribution and reproduction in any medium or format, as long as you give appropriate credit to the original author(s) and the source, provide a link to the Creative Commons licence, and indicate if changes were made. The images or other third party material in this article are included in the article's Creative Commons licence, unless indicated otherwise in a credit line to the material. If material is not included in the article's Creative Commons licence and your intended use is not permitted by statutory regulation or exceeds the permitted use, you will need to obtain permission directly from the copyright holder. To view a copy of this licence, visit http://creativeco mmons.org/licenses/by/4.0/. The Creative Commons Public Domain Dedication waiver (http://creativecommons.org/publicdomain/ zero/1.0/) applies to the data made available in this article, unless otherwise stated in a credit line to the data. 
requires the procedure of plant tissue culture and usually takes a long period to accumulate a large number of mutants' collections.

FNB uses high-energy neutrons to irradiate plant seeds and mainly induces random deletions of various sizes on chromosomes. Because deletions likely cause either complete/partial loss of the corresponding genome fragments or induce frame-shift mutations, FNB is a very powerful mutagen to generate null mutations. In addition, the background mutations are much less in FNB mutants as compare to the EMS mutants, making it easier for further causative gene cloning work. Hence, FNB has been broadly used in creating mutant libraries in many model and crop plants, including Arabidopsis thaliana, M. truncatula, rice (Oryza sativa), and soybean (Glycine max) [5-7].

Despite the efficiency of FNB in mutating plant genomes, it is still challenging to identify the mutation sites. The classical positional-cloning method certainly could locate the candidate gene, it requires a segregating population derived from the intercross between the FNB mutant and another accession, and complex genetic linkage analysis for the molecular markers with mutation site $[8,9]$. Consequently, it could take years to identify the candidate mutation $[8,9]$. To accelerate the process of mutation identification, few new methods including CGH and Deletion-TILLING (De-TILLING) have been developed [6, 10]. In addition, Ge et at. (2016) reported a successful case using the Affymetrix microarray-based expression profiling dataset to identify the causative DNA deletion in M. truncatula [11]. These new methods were principally based on the DNA fragment deletion of substantial size that could lead to the decrease of DNA hybrid signal compared with wild-type or down-regulation of few adjacent genes, the limitation mainly lies in the relatively low resolution and accuracy. Along with the development of next-generation sequencing technology, several new algorithms/tools have been developed to detect DNA fragment deletions of various sizes, such as Pindel [12], BreakDancer [13, 14] and FNBtools [15]. FNBtools is particularly specialized for FNB mutants. It took advantage of the CIGAR information from the informative reads extracted from the short reads alignment files to locate the DNA breaking point and used a segregating population to detect the linkage between the deletion and the mutant phenotype.

These new methods continually improve the accuracy and reliability in predicting the structural variations on chromosomes. However, due to the complexity of genomes, particularly the duplications resulted from the whole genome duplication, we found that it is still challenging in detecting reliable mutations in many cases. In some cases, the reported deletions reflected false positives. In this work, we hypothesized that small deletions in FNB mutants can be viewed as polymorphic sites between mutants and wild-type plants, while large-size deletions can be viewed as presence-absence variations (PAV), which typically show extremely low or even no reads coverage in mutants, in contrast to the parallel datasets of controls which should display normal reads coverage. Based on this hypothesis, we developed a straightforward pipeline to detect the mutation sites of FNB mutants by combining the variant-calling pipeline and customized filtering of the variants. To establish the linkage between the identified mutation and the mutant phenotype, we applied the pipeline to the homozygous mutant and wild-type like plants derived from an $F_{2}$ population and identified the mutated gene responsible for the phenotype.

\section{Result}

\section{The pipeline for calling and filtering deletions of FNB mutants in $\mathrm{M}$. truncatula}

In order to identify the associated deletions linked to the mutants' phenotypes, the mutants are usually backcrossed to the wild-type plants. Backcrossing not only purifies the background, but also creates a segregating population that can be used for Bulked Segregant Analysis coupled with Whole-Genome Sequencing (BSA-Seq) $[16,17]$. For BSA-Seq, if the $\mathrm{F}_{1}$ plants show wild-type like phenotype and the ratio of mutant to wild-type like phenotype is $1: 3$ in $F_{2}$ progeny, then the mutant is likely to be recessive. In this scenario, the mutant plants are pooled for DNA sequencing. The wild-type like plants, which are a mixture of both homozygous and heterozygous genotype, are also pooled for DNA sequencing. If $\mathrm{F}_{1}$ plants do not show wild-type phenotype and the ratio of mutant phenotype to wild-type like phenotype is significantly away from 1:3, the mutant is likely not to be recessive. In this scenario, $\mathrm{F}_{3}$ generation is used to identify homozygous mutant and wild-type like plants, which do not display phenotypic segregating in $\mathrm{F}_{3}$ progeny. The homozygous mutants are pooled for sequencing, as well as the wild-type like plants.

In case, if urgent identification of candidate deletions is needed and segregating population is not available, the individual FNB mutant plants are directly pooled and sequenced. In addition, the wild-type plants are also pooled and sequenced.

To achieve adequate coverage, we propose at least a $20 \times$ depth of the Illumina paired-end sequencing. After the quality control procedures for the raw reads, a variant discovery calling pipeline is used to identify the mutations of the FNB mutants (Figs. 1 and 2). We recommend the GATK pipeline for a parallel calling for a cohort of independent FNB mutants simultaneously, which would 


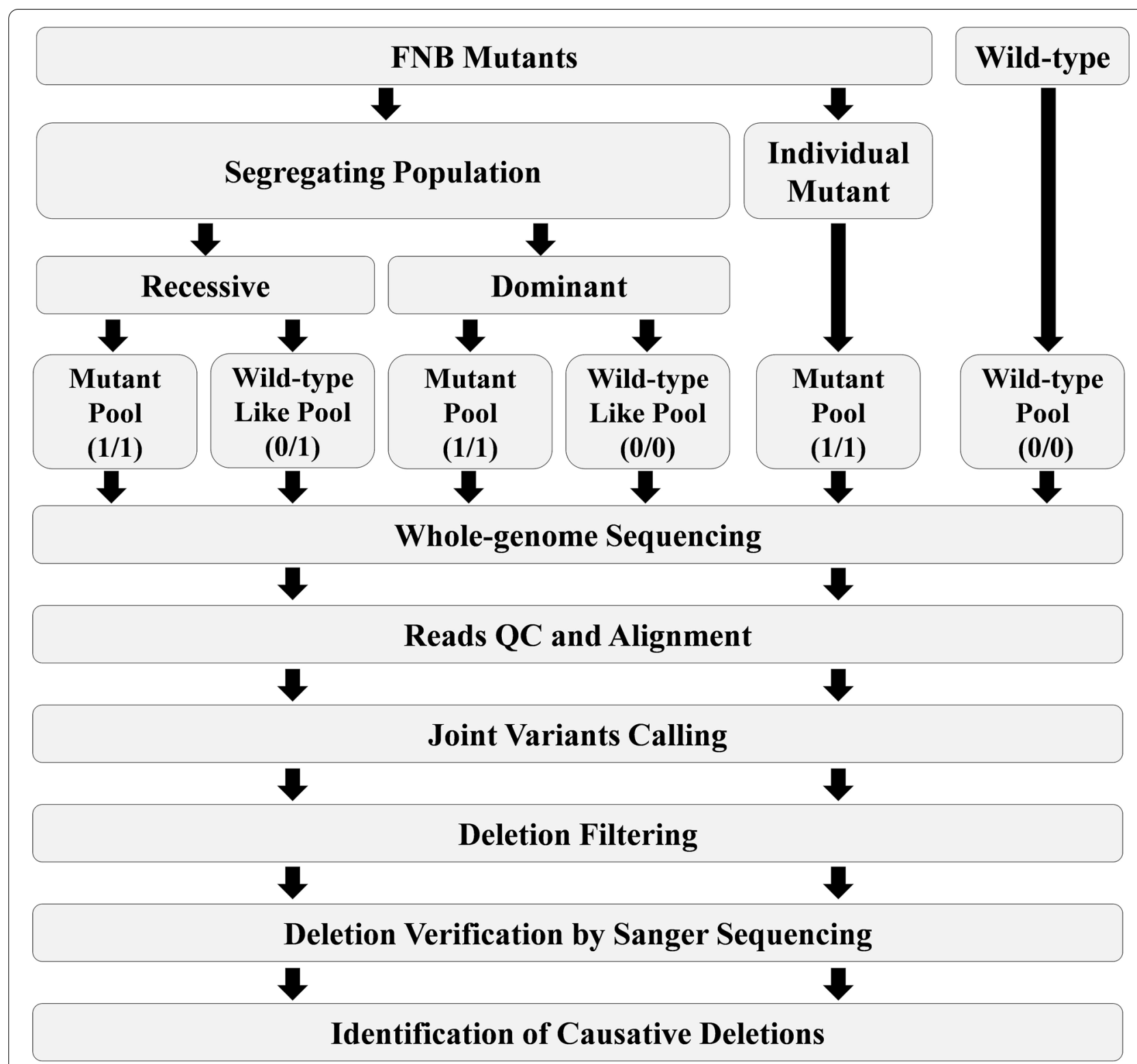

Fig. 1 The step-by-step workflow diagram to identify candicate causative deletions for FNB mutants. FNB mutants, either from a segregating population or individual mutant, were sequenced by NGS. The wild type plants, and the wild-type like plants from the same segregating population of FNB mutants were sequenced as well. After the joint variants calling and fitering, deletions were verified by Sanger sequencing, and the causative deletion was identified. The numbers in the brackets represet the genotypes (0/0: homozygous wild type; 0/1: heterozygous; 1/1: homozygous mutant)

contribute to filter the background mutations [18-21]. If the mutant is recessive, the genotype of the mutant pool is a homozygous mutation, whereas the genotype of the corresponding wild-type like plants pool is heterozygous mutation. In contrast, wild-type plants and other irrelevant mutants are all wild-type genotype. If the mutant is not recessive, the mutant pool is a homozygous mutation, and wild-type pool, which is made of the wild-type like plants that do not show phenotypic segregating in
$\mathrm{F}_{3}$ generation, is a homozygous wild-type genotype. The wild-type plants and other irrelevant mutants are also homozygous wild-type genotype. For the individual mutant, the mutant pool is a homozygous mutation, whereas all other pools and wild-type plants are wildtype genotypes. Based on these criteria, the variants are filtered for each mutant. The deletions that pass the filtering are further compared with the genome annotation file. If the mutations fall within the gene coding regions, 


\section{WGS Reads Alignment and Deletion Calling}

b

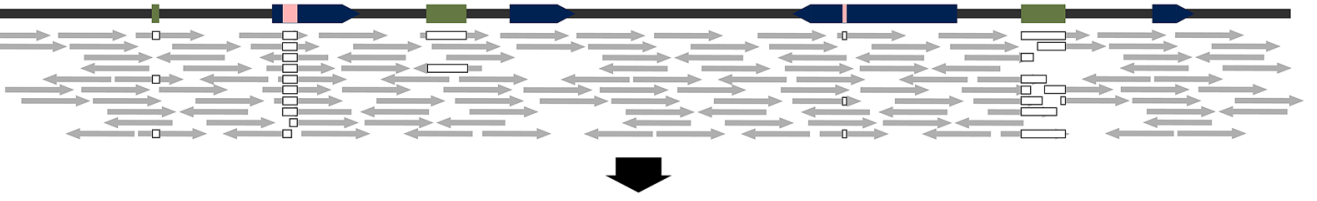

Segregating Population

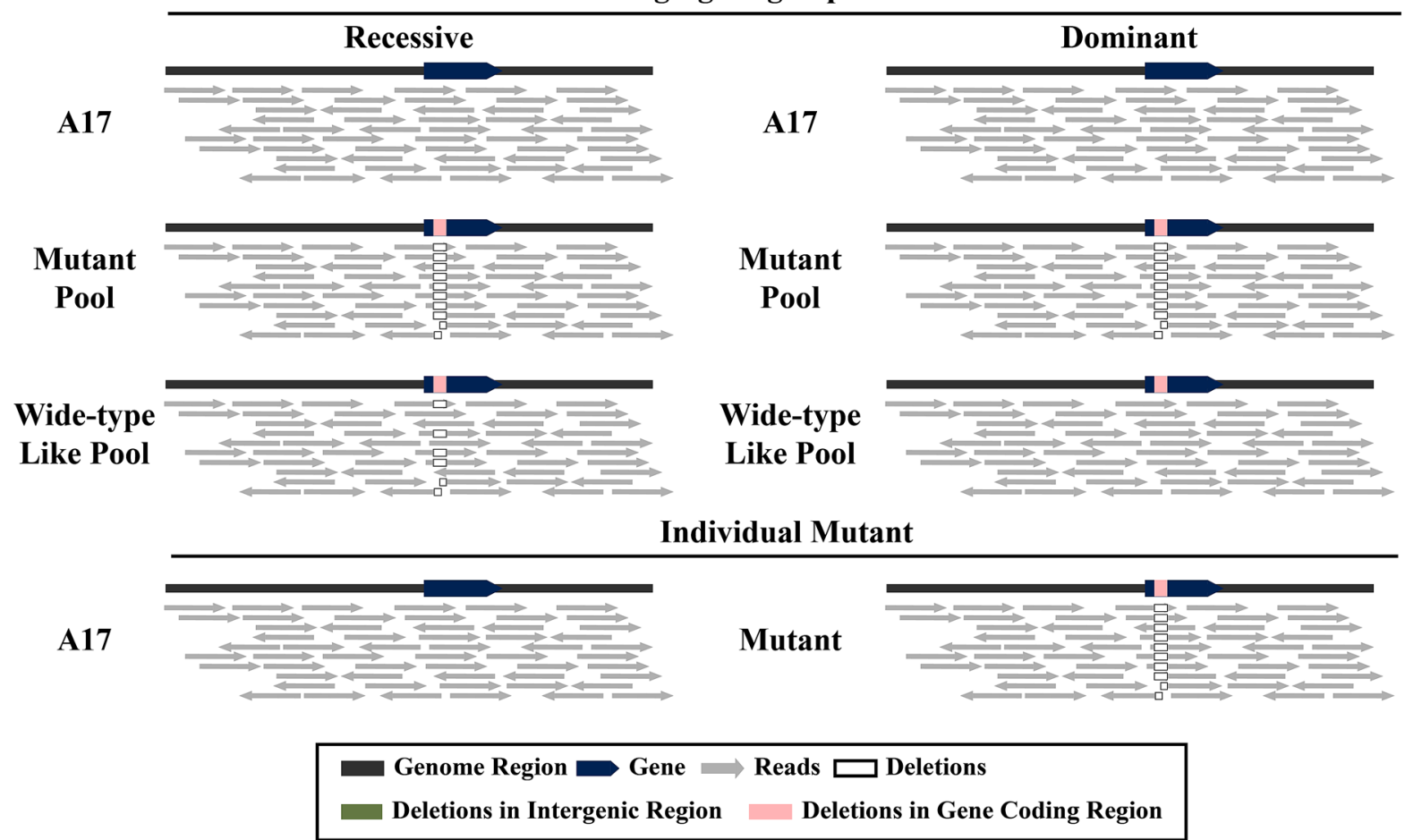

Fig. 2 The pipeline of calling and filtering deletions. a Illustration of the reads alignment and deletion calling steps for FNB mutant NGS data. $\mathbf{b}$ Identification of causative deletions for FNB mutants from segregating populations. $\mathbf{c}$ Identification of candidate deletions for individual FNB mutant

they can be considered candidate mutations. Additionally, the short reads alignment files, such as BAM files, are used to visually confirm the mutations. Finally, the candidate deletion borders are amplified and further confirmed by Sanger sequencing.

\section{A case study, identification of the causative gene for an FNB mutant using FNB-BSA-Seq in M. truncatula}

To evaluate the effectiveness of the pipeline and identify the causative gene for a fixation-minus $\left(f^{-} x^{-}\right.$FNB mutant, which developed white and small nodules, named as white nodule (wn) (Fig. 3a, b), we applied BSASeq pipeline to the $w n$ mutant. $w n$ was first backcrossed to wild-type $M$. truncatula (cv. Jemalong A17). In the $\mathrm{F}_{2}$ generation, 14 mutant plants that developed fix ${ }^{-}$nodules and 46 wild-type like plants that produced normal nodules were observed. The chi-square test indicated that the mutant to wild-type segregation ratio fits 1:3, suggesting that $w n$ is a single recessive mutation. The mutant plants were all pooled for WGS. As the control, 25 wild-type like plants were also pooled for WGS, as well as the wildtype plant A17 that can serve as an additional control. For the mutant pool, more than 42.6 million paired-end reads $(2 \times 150 \mathrm{bp})$ were produced, which represents a $29.7 \times$ sequencing depth (Table 1 ). For the wild-type like plants pool and A17 pool, 48.8 and 43.1 million reads were produced, representing $34.1 \times$ and $30.1 \times$ sequencing depth respectively (Table 1 ). The reads were mapped to the latest reference genome of $M$. truncatula (MtrunA17r5.0) [22] and the cohort polymorphisms were called using the mapped reads. Since the mutant is recessive, the causative mutation must be homozygous deletion, whereas the pool made of the wild-type like plants should be heterozygous genotype, and A17 plants should be wild-type genotype. On the basis of this guideline, the identified deletions were further filtered. In the mutant pool, totally nineteen homozygous deletions, spanning one to eighteen base pairs, were found, and the 


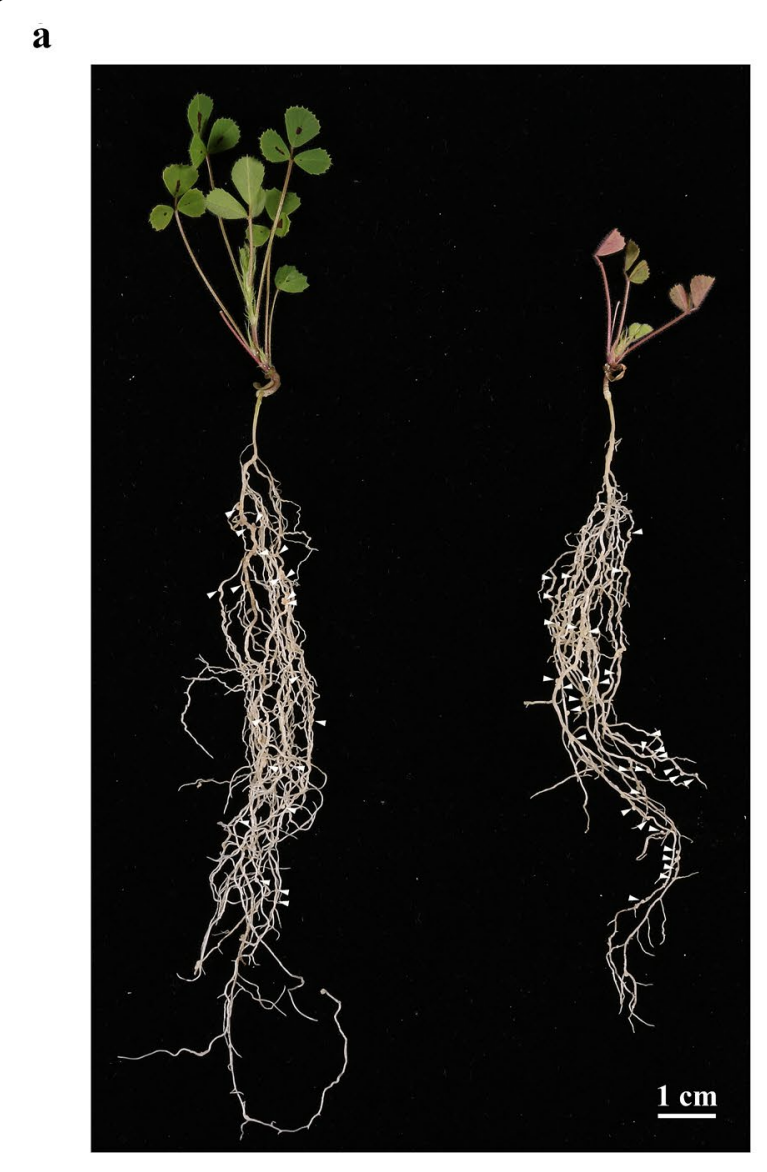

c

\section{MtrunA17_Chr3g0119041/SymCRK}
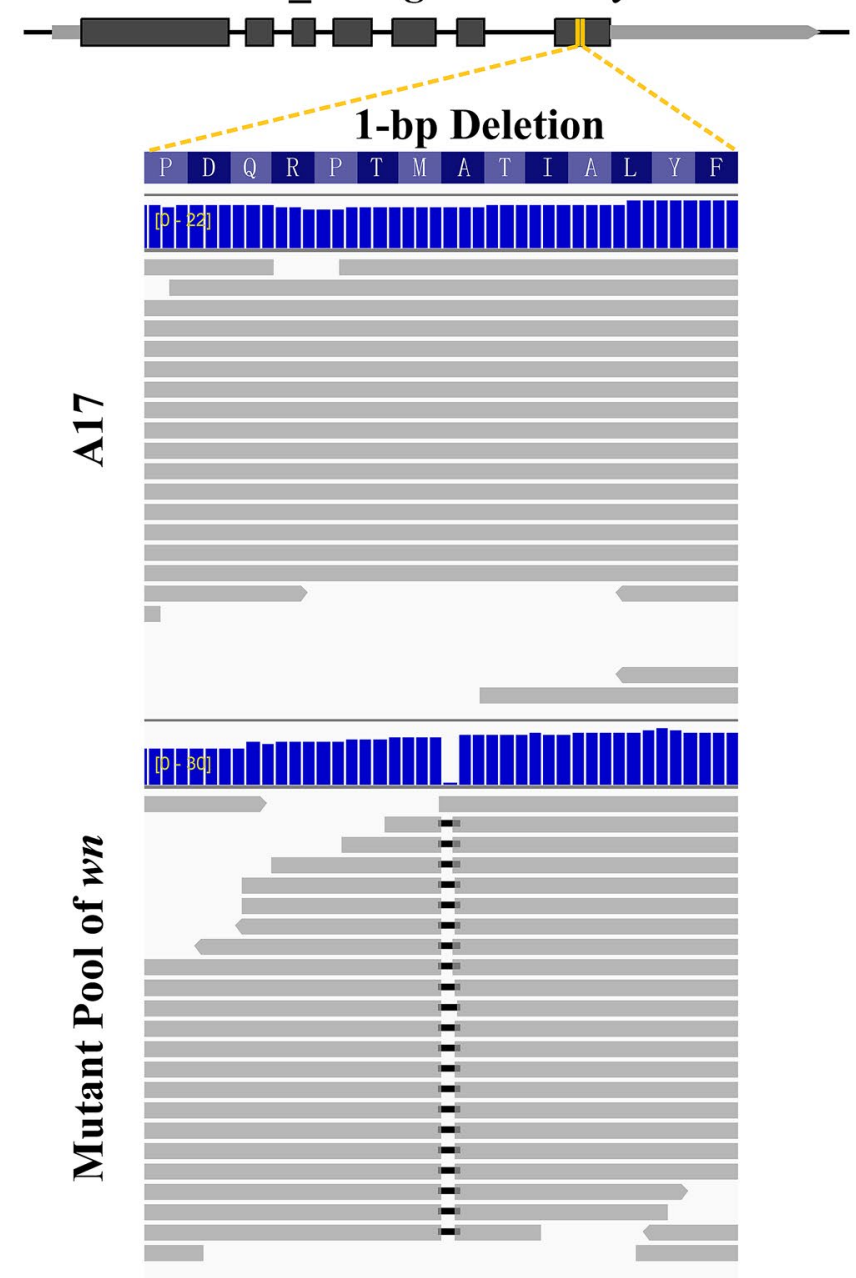

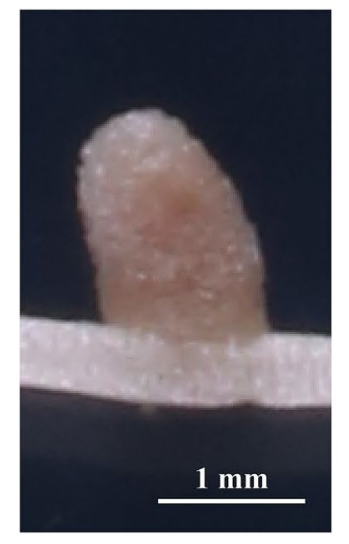

A17

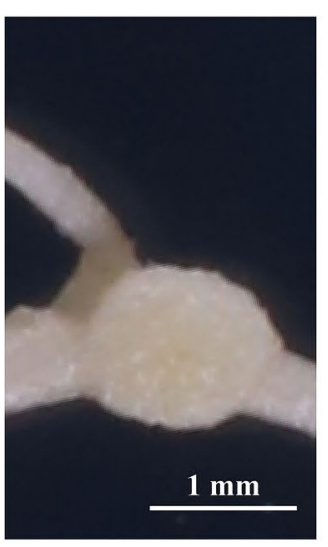

wn

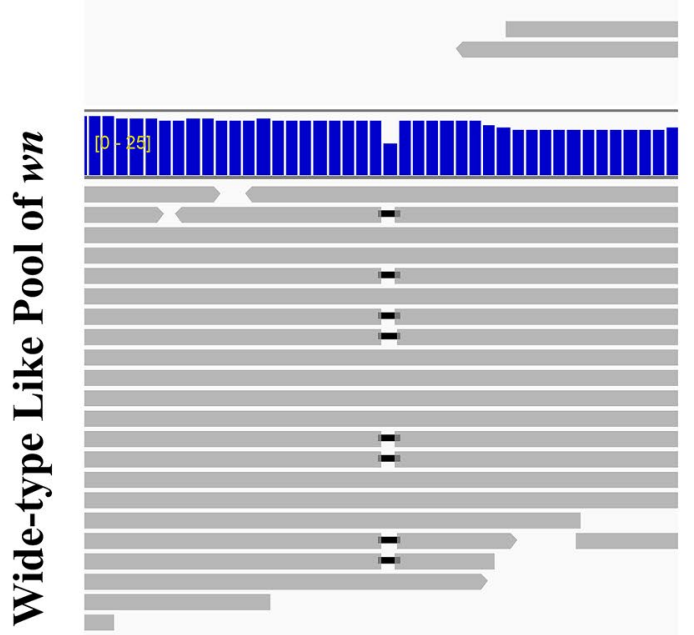

Fig. 3 The phenotype and reads alignment of wn mutant. a The phenotype of whole plants for A17 (left) and wn mutant (right). b The nodule phenotype for A17 (left) and wn mutant (right). c The reads alignment for A17, mutant pool of wn and wide-type like pool of wn 
Table 1 Statistics of short reads and sequencing depth

\begin{tabular}{lllll}
\hline & A17 & $\begin{array}{l}\text { wn Mutant } \\
\text { Pool }\end{array}$ & $\begin{array}{l}\text { wn Wild- } \\
\text { type Like } \\
\text { Pool }\end{array}$ & yl1 \\
\hline $\begin{array}{l}\text { Reads number } \\
\begin{array}{l}\text { Sequencing } \\
\text { depth }\end{array}\end{array}$ & $\begin{array}{l}43,067,006 \\
\text { 30.1x }\end{array}$ & $\begin{array}{l}42,571,812 \\
29.7 x\end{array}$ & $\begin{array}{l}48,823,767 \\
34.1 x\end{array}$ & $\begin{array}{l}57,358,177 \\
40.1 \times\end{array}$ \\
\hline
\end{tabular}

corresponding genotypes of the wild-type like plants pool and A17 were heterozygous and wild type, respectively.
The deletions were further visually confirmed by visualizing the short reads alignment details in Integrative Genomics Viewer (IGV) [23]. Among the nineteen deletions, sixteen are located in the intergenic regions, and two are located in the untranslated regions (UTRs) and do not affect the coding sequences. The only one located in the coding region is a single base pair deletion, which caused the frameshift of MtrunA17 Chr3g0119041 (Fig. 3c and Additional file 2: Table S1). The deletion was further confirmed by Sanger sequencing (Fig. 4a). MtrunA17_Chr3g0119041 encodes a Serine/Threonine Kinase (STK), also termed

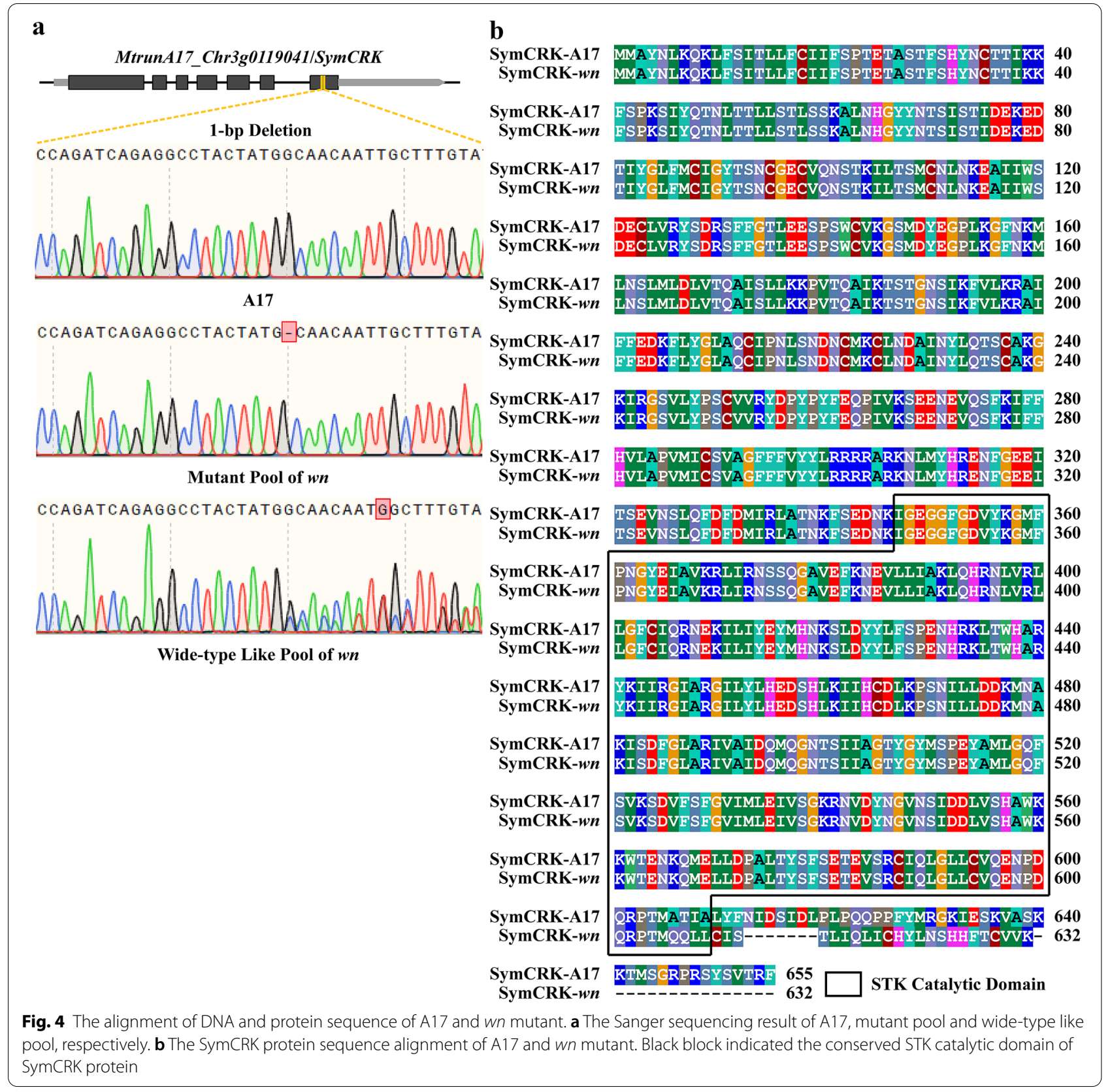


as SymCRK in a previous report [24] (Fig. 4b). It has been documented that SymCRK controls the senescence process of nodules in $M$. truncatula. The deletion in the coding region of SymCRK caused the defect in the STK catalytic domain. Similar to $w n$ mutant, symcrk mutant also produced white and necrotic nodules [24]. These results suggested that 1-bp deletion in SymCRK caused the defective nitrogen fixation phenotype of $w n$ mutant, reflecting that $S y m C R K$ was the causative gene of $w n$ mutant.
A case study, identification of the causative deletion for an individual FNB mutant in M. truncatula

To evaluate the effectiveness of the pipeline for individual FNB mutants, we applied the pipeline to an FNB individual mutant, which developed yellow leaves and was named yellow leaves 1 (yll). Phenotypic analysis indicated that the chlorophyll concentration of $y l 1$ mutant was significantly lower than A17 plants, resulting in yellow-green cotyledon and leaves (Fig. 5a).

To rapidly identify the possible causative gene for $y l 1$, $y l 1$ plants were pooled and directly sequenced by WGS. Totally 57.4 million paired-end reads were produced for $\mathbf{a}$
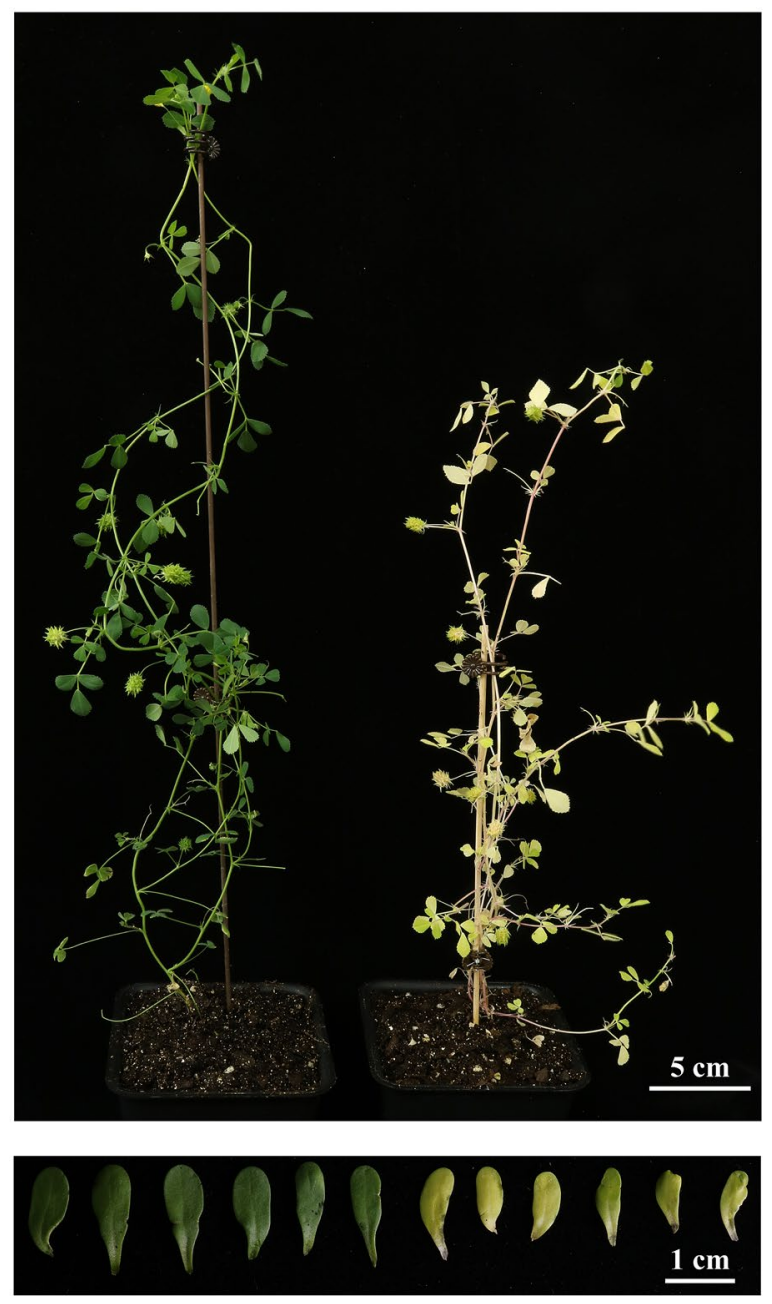

A17 b
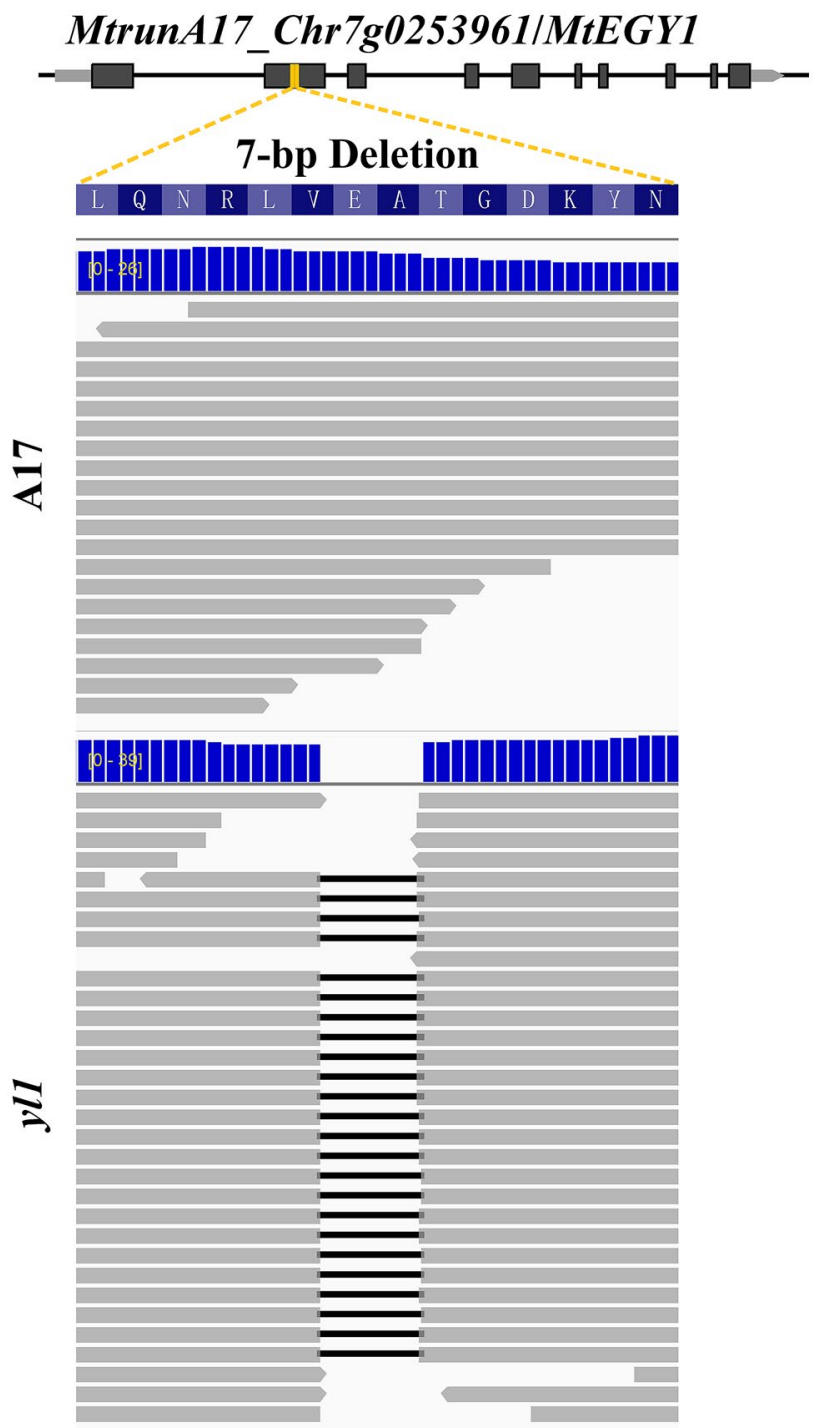

Fig. 5 The phenotype and reads alignment of y/1 mutant. a The phenotype of whole plants and cotyledon for A17 (left) and y/1 mutant (right). b The reads alignment for $\mathrm{A} 17$ and $y / 1$ mutant 
$y l 1$, representing a $40.1 \times$ sequencing depth (Table 1 ). For polymorphisms calling, both $y l 1$ and A17 pools were called simultaneously. The raw polymorphism data were first filtered by the quality, followed by the genotype. In the filtering process, we detected a large deletion which is about one kilobase pair (kb). This large deletion presents in both $y l 1$ and A17, thus it was not considered a candidate deletion (Additional file 1: Fig. S1). The deletions that were only presented in $y l 1$ pool were considered potential candidates. In total, 115 deletions existed in $y l 1$ pool only. Among these deletions, two were located in the coding region of genes on chromosome 7 , and caused the frameshift mutation for the corresponding genes (Additional file 3: Table S2).

Visualization of the short reads alignment files in IGV indicated that the candidate deletions were well supported by the reads alignment (Fig. 5b). One of the candidate deletions is located in MtrunA17 Chr7g0223481, which encodes a putative stigma-specific protein. The 1-bp deletion caused the frameshift of MtrunA17_Chr7g0223481. MtrunA17_Chr7g0223481 is the homolog of At1g50650 in Arabidopsis thaliana, which encodes the STIG1 family peptide KERBEROS (KRS). KRS regulates the development of embryo sheath [25], and krs mutant showed the absence of sheath production by endosperm [25]. Another deletion was located in the second exon of MtrunA17_Chr7g0253961, which encodes a putative peptidase M50 protein (Fig. $5 \mathrm{~b}$ ). The 7-bp deletion caused frameshift mutation in MtrunA17_ Chr7g0253961 and the loss of S2P/M50 (Site-2 protease/zinc metalloproteases) domain, which is critical for the proper function of the protein (Fig. 6a, b). Based on the homolog analysis, we found that the MtrunA17_ Chr7g0253961 is the homolog of At5g35220 in A. thaliana, which encodes ETHYLENE-DEPENDENT

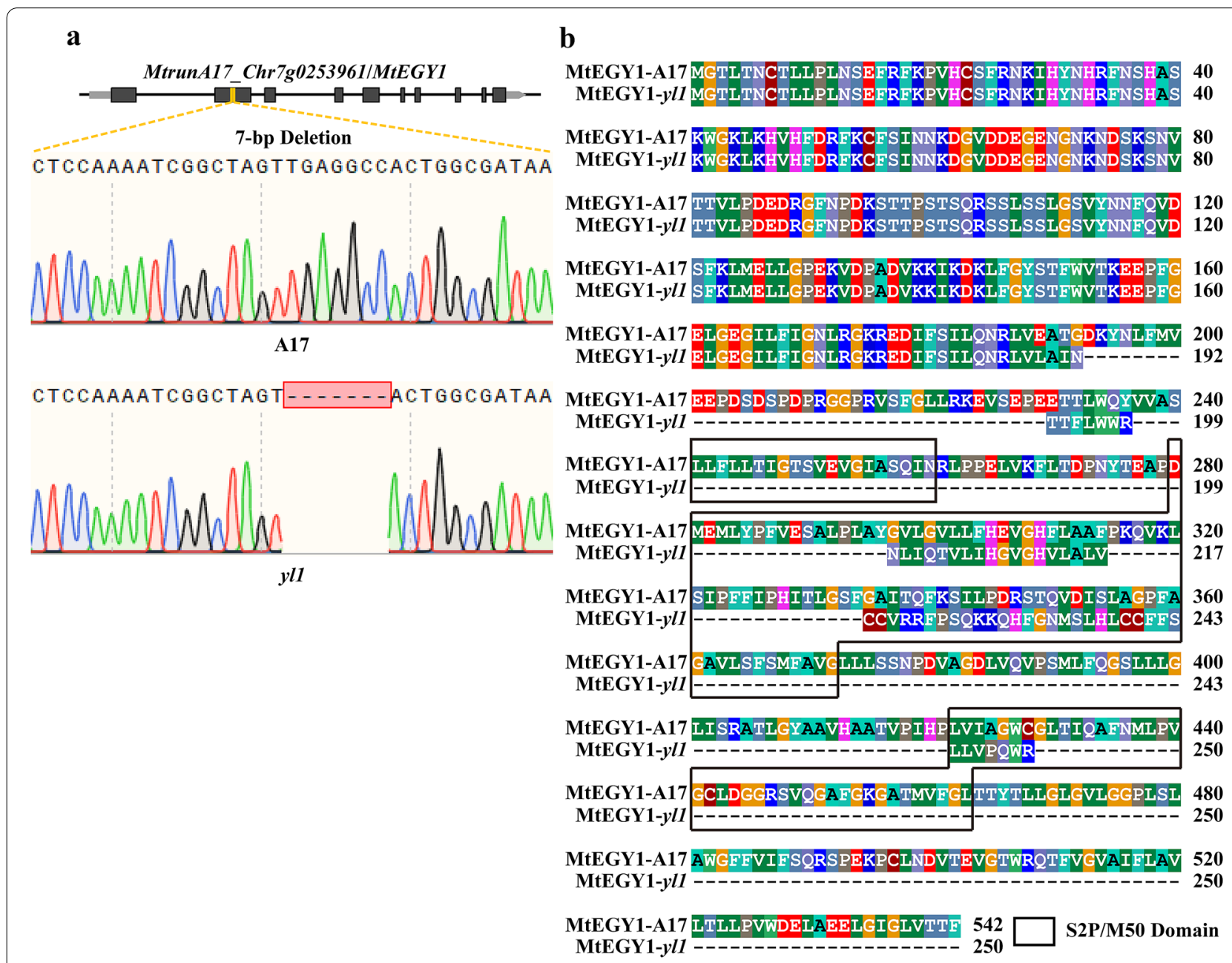

Fig. 6 The alignment of DNA and protein sequence of A17 and $y / 1$ mutant. a The Sanger sequencing result of A17 and y/1 mutant. b The alignment of MtEGY1 from A17 and y/1 mutant. Black block indicated the conserved S2P/M50 domain of MtEGY1 protein 
GRAVITROPISM-DEFICIENT AND YELLOW-GREEN (EGY1). A previous report documented that EGY1 is required for the development of chloroplast and accumulation of chlorophyll in Arabidopsis [26]. The A. thaliana egy1-1 mutant developed yellow-green leaves, which similar to the leaves of $y l 1$ mutant. Thus, it is highly likely that the EGY1 homologous gene, MtrunA17_ Chr7g0253961, is the causative gene of $y l 1$.

\section{Discussion}

Mutagenesis is a very important tool to dissect genes' function in plant genetic research. FNB, which employs high-energy neutron as the mutagen, is very effective in generating various deletions on the chromosomes. Owing to its mutagenesis efficiency in generating null mutations, FNB has long been used to create large-scale genetic resources for forward and reverse genetics studies, and even used as a breeding technique for many species. Due to the complexity of plant genomes, identifying the causative deletions for FNB mutants has always been challenging in many studies, particularly before the era of NGS. Positional cloning was one of the most popular methods to identify the causative gene for FNB mutants of interest. However, it was rather time-consuming. In order to increase the efficiency in identifying the mutations, new approaches such as CGH and Deletion-TILLING have been developed [6, 27]. These new approaches provided new solutions in dealing with the FNB mutants. However, the effectiveness and accuracy are still to be improved for such probe or PCR-based methods [15]. These disadvantages have limited the utilization of FNB mutants as an invaluable genetic resource. The rapid progressing of NGS technology has greatly renovated the strategy in detecting the whole genome polymorphisms and offers many advantages over the traditional methods in identifying the mutations for FNB mutants. With continuous cost reduction, NGS has been a regular tool and accessible to most researchers. WGS by NGS for FNB mutants not only increases the sensitivity in detecting mutations on chromosomes, but also improves the reliability and resolution in identifying deletions. In this work, we developed a useful and straightforward pipeline for the identification of candidate deletion for FNB mutants in M. truncatula. The pipeline takes full advantage of high-depth WGS and advanced deletion-calling algorithm and presents a viable solution in identifying the candidate causative genes for the FNB mutants.

The pipeline developed by this work is effective in identifying the causative deletions for FNB mutants, as demonstrated by the application of pipeline to the two representative FNB mutants, one in a segregating population and another in individual FNB mutant. FNB-BSASeq has detected a single base pair deletion in SymCRK coding sequence of $w n$ mutant. The 1-bp deletion in $w n$ mutant caused frameshift null mutation of SymCRK and led to white and necrotic nodules, which resembles the phenotype of symcrk mutant. The 1-bp deletion is rather small and would not be identified by the probe-based approaches. Combining $30 \times$ deep sequencing and deletion calling/filtering pipeline, the 1-bp deletion of $w n$ mutant stood out with high-quality scores in the report of the pipeline, demonstrating that the pipeline is very effective in identifying small deletions of FNB mutant.

For $y l 1$ mutant, there is no segregating population, thus we are unable to establish the linkage between the deletion and mutant. However, a mutant without a useful segregating population is very common in the practices of genetic researches, as generating a segregating involves multiple steps and progenies, and usually takes a long time, especially for those plants with long life cycles (M. truncatula typically has a life cycle from four to six months). It would be very useful if the candidate deletions could be identified for the individual FNB mutant. In our practice, the pipeline reported only two candidate deletions that are located in the coding region of genes. This is probably because that FNB mutant usually has a less background mutation compared with the mutants induced by other chemical mutagens like ethyl methanesulfonate (EMS) [6]. The two candidate deletions of $y l 1$ mutant are 1-bp and 7-bp respectively, both causing the frameshift in the coding sequences. Through protein homologs and annotation searches, we found that the deletion in MtrunA17_Chr7g0223481 is likely the causative mutation of $y l 1$, since MtrunA17_Chr7g0223481 is the homolog of Arabidopsis EGY1, whose mutation led to the similar phenotype of $y l 1$. Taken together, the example of $y l 1$ mutant demonstrated not only the effectiveness of the pipeline in identifying the deletion for the FNB mutants, but also the possibility of application of the pipeline to the individual FNB mutant for causative gene cloning.

WGS uses high coverage short reads to examine the homogeneity between the reference genome and short reads. Sequencing depth is an important parameter that determines the sensitivity and reproducibility in detecting variations among genomes $[28,29]$. In this work, we proposed a minimum of $20 \times$ sequencing depth for mutation detection. Given sufficient sequencing depth, WGS could theoretically detect all potential deletions caused either by artificial mutagenesis or natural variation, which has driven the utilization of WGS for indel calling in many studies. WGS usually generates a large-scale dataset including tens of millions of raw short reads, which require well-designed algorithms and intense data analyzing procedures to extract the polymorphic information. During the past decade, there are a few algorithms 
developed to detect the deletions from the WGS data [12-15]. These algorithms/tools investigate the deletions on chromosomes from different angles, including using informative reads to calculate the deletions or reads coverage survey. In this work, we treated the small deletions of FNB mutant as the indel sites, which widely exist in the natural populations and have been tensely studied in recent years. There have been successful tools developed to identify such small indels, including GATK4's HaplotypeCaller. Although it is not designed for FNB mutants, HaplotypeCaller performs well in terms of sensitivity and accuracy, possibly owing to its unique ability of denovo local assembly of haplotypes for the regions where a potential polymorphism occurs [21]. GATK4's variant discovery workflow is well documented and maintained by Broad Institute. Applying this workflow to the WGS dataset of FNB mutants is straightforward and requires a minimal learning process, making it feasible for most researchers to rapidly identify the candidate deletions for FNB mutants.

\section{Conclusion}

Despite the progress in developing methods to identify deletions for FNB mutants, it remains challenging to rapidly isolate the causative mutations in many studies. Combining WGS, the variant discovery workflow, and filtering by comparing the genotype of FNB mutants and control lines, we developed a simple but efficient pipeline to rapidly identify the candidate causative deletions for FNB mutants in M. truncatula. As demonstrated by the two case studies, the pipeline combines sensitivity and accuracy in detecting mutations, and the filtering process by comparing with multiple controls is very useful to pinpoint the causative mutations. FNB mutant collections are available for many model and crop species. Due to the difficulty in rapidly identifying the causative genes, these invaluable resources have not been fully utilized. The principle and pipeline described here can also be applied to the FNB mutants of other species, offering a reliable solution to utilize FNB mutants for genetic research or crop breeding.

\section{Methods}

\section{Plant materials}

The M. truncatula plants were germinated in a petri dish and placed at $4{ }^{\circ} \mathrm{C}$ for one week. After germination, the plants were grown in a growth chamber at $22{ }^{\circ} \mathrm{C} / 16 \mathrm{~h}$ light and $20{ }^{\circ} \mathrm{C} / 8 \mathrm{~h}$ dark. To generate the $\mathrm{F}_{2}$ segregating population, $w n$ mutant was backcrossed to $M$. truncatula cv. Jemalong A17 and $F_{1}$ plants were selfed to generate the $F_{2}$ population. The cotyledon and leaf phenotypes of $y l 1$ and A17 were analyzed one month and two months post-germination respectively.

\section{Rhizobia inoculation and nodule phenotype analysis}

The $\mathrm{F}_{2}$ population of $w n$ mutant was grown in sand and inoculation with rhizobia Sm2011 (Sinorhizobium meliloti 2011) as previously reported [30]. The nodules of wide-type and $w n$ were observed and analyzed with the stereomicroscope after 30 days post-inoculation. The plants with white and necrotic nodules were pooled as the mutant pool, and the plants with red and normal nodules were pooled as the wild-type like pool.

\section{DNA extraction and sequencing}

The DNA samples from leaves were extracted using the Trelief ${ }^{\mathrm{TM}}$ Plant Genomic DNA Kit (Beijing TsingKe Biotech; TSP101). The DNA samples were sequenced on an Illumina Nova-seq platform with a 150 -bp pairedend (PE) protocol.

\section{Sequence alignment, indel calling, and visualization}

The polymorphism calling was conducted according to GATK4's (GATK v4.1.7.0) instruction [19]. Briefly, the 150-bp pair-end reads were mapped to the $M$. truncatula reference genome (MtrunA17r5.0) using the BWA-MEN (Burrows-Wheeler Aligner; Version 0.7.12-r1039) [31]. For reads alignment, mem algorithm was used with a minimum seed length of 19 , band width of 100, and off-diagonal X-dropoff of 100. After sorting, adding reads groups, validating, and marking for duplicates, the mapped reads in bam format were used to call variants by HaplotypeCaller in GVCF mode [20]. For the calling step, the assembly-region-padding was set to 100, the base-quality-score-threshold was set to 19 , the max-reads-per-alignment-start was set to 50 , and the max-assembly-region-size and min-assemblyregion-size were set to 300 and 50 , respectively. The individual GVCF files were subjected to joint variant calling. The indels were filtered from the variants after calling using SelectVariants function of GATK. The deletions were selected from the indels for further analysis. The IGV (v2.8.2) software was used for further visual verification of deletions of the mapped reads [23].

\section{Sanger sequencing validation}

The deletion borders of SymCRK in $w n$ and MtEGY1 in $y l 1$ were amplified using $\mathrm{Q} 5{ }^{\circledR}$ High-Fidelity DNA Polymerase (NEB \#M0491) with primers spanning the target deletion sites. The PCR was conducted as follows: $98{ }^{\circ} \mathrm{C}$ for $10 \mathrm{~s}, 60{ }^{\circ} \mathrm{C}$ for $30 \mathrm{~s}$ and $72{ }^{\circ} \mathrm{C}$ for $30 \mathrm{~s}$ for 35 cycles. The PCR products were Sanger sequenced. The primers were listed in the Additional file 4: Table S3. 


\begin{abstract}
Abbreviations
FNB: Fast neutron bombardment; CGH: Comparative genomic hybridization; CNVs: Copy number variations; WGS: Whole-genome sequence; EMS: Ethyl methane sulfonate; T-DNA:Transfer DNA; De-TILLING: Deletion-TILLING; BSAseq: Bulked segregant analysis coupled with whole-genome sequencing; IGV: Integrative genomics viewer; NGS: Next-generation sequencing.
\end{abstract}

\section{Supplementary Information}

The online version contains supplementary material available at https://doi. org/10.1186/s13007-021-00765-y.

Additional file 1: Figure $\mathbf{S 1}$ The identified large fragment deletion of $y / 1$ mutant. (a) The coordinate and size of the large deletion identified in both A17 and $y / 1$ mutant. (b) Visualization of aligned reads surroungding the deletion in both $\mathrm{A} 17$ and $y / 1$ mutant

Additional file 2: Table S1. The potential deletions in wn mutant. The details of potential deletions in wn mutant populations were listed, which include the location, sequence, length, genotype in different samples and the mutant type of deletions.

Additional file 3: Table S2. The potential deletions in $y / 1$ mutant. The details of potential deletions in $y / 1$ mutant populations were listed, which include the location, sequence, length, genotype in different samples and the mutant type of deletions.

Additional file 4: Table S3. The list of primers. The details of primers for genotyping of SymCRK and MtEGY1 were listed.

\section{Acknowledgements}

The authors thank Dr. Intikhab Alam for critical reading of the manuscript.

\section{Authors' contributions}

$H D, Z J$, and LG conceived, designed, and conducted the research. JL and WH contributed to the research and revised the manuscript. HD and ZJ analyzed data. LG wrote the manuscript. All authors read and approved the final manuscript.

\section{Funding}

This work was funded by the National Science Foundation of China (NSFC, Project 31771345 and 32070305) and the startup fund provided to Ge's lab by South China Agricultural University. Funding bodies were not involved in the design of the study; collection, analysis, interpretation of data, and writing the manuscript.

\section{Availability of data and materials}

The raw NGS data generated in this study are deposited into NCBI database under the accession number PRJNA714779.

\section{Declarations}

\section{Ethics approval and consent to participate}

Not applicable.

\section{Consent for publication}

Not applicable.

\section{Competing interests}

The authors declare that they have no competing interests.

\section{Author details}

${ }^{1}$ Guangdong Key Laboratory for Innovative Development and Utilization of Forest Plant Germplasm, College of Forestry and Landscape Architecture, South China Agricultural University, Guangzhou 510642, Guangdong, China. ${ }^{2}$ Guangdong Engineering Research Center for Grassland Science, South China Agricultural University, Guangzhou, China. ${ }^{3}$ State Key Laboratory for Conservation and Utilization of Subtropical Agro-Bioresources, College of Life Sciences,
South China Agricultural University, Guangzhou 510642, China. ${ }^{4}$ The Guangdong Subcenter of the National Center for Soybean Improvement, College of Agriculture, South China Agricultural University, Guangzhou 510642, China.

Received: 21 March 2021 Accepted: 9 June 2021

Published online: 16 June 2021

\section{References}

1. Alonso JM, Stepanova AN, Leisse TJ, Kim CJ, Chen H, Shinn P, Stevenson DK, Zimmerman J, Barajas P, Cheuk R, et al. Genome-wide insertional mutagenesis of Arabidopsis thaliana. Science. 2003;301(5633):653-7.

2. Sha Y, Li S, Pei Z, Luo L, Tian Y, He C. Generation and flanking sequence analysis of a rice T-DNA tagged population. Theor Appl Genet. 2004;108(2):306-14.

3. Kaul ML, Bhan AK. Mutagenic effectiveness and efficiency of EMS, DES and gamma-rays in rice. Theor Appl Genet. 1977:50(5):241-6.

4. Khadr FH, Shukry MW. Quantitative variability in wheat following irradiation, EMS, and hybridization. Theor Appl Genet. 1972;42(4):174-80.

5. Bruggemann E, Handwerger K, Essex C, Storz G. Analysis of fast neutrongenerated mutants at the Arabidopsis thaliana HY4 locus. Plant J. 1996;10(4):755-60.

6. Rogers C, Wen J, Chen R, Oldroyd G. Deletion-based reverse genetics in Medicago truncatula. Plant Physiol. 2009;151(3):1077-86.

7. Bolon YT, Haun WJ, Xu WW, Grant D, Stacey MG, Nelson RT, Gerhardt DJ, Jeddeloh JA, Stacey G, Muehlbauer GJ, et al. Phenotypic and genomic analyses of a fast neutron mutant population resource in soybean. Plant Physiol. 2011;156(1):240-53.

8. Chen J, Yu J, Ge L, Wang H, Berbel A, Liu Y, Chen Y, Li G, Tadege M, Wen J, et al. Control of dissected leaf morphology by a Cys(2)His(2) zinc finger transcription factor in the model legume Medicago truncatula. Proc Natl Acad Sci USA. 2010:107(23):10754-9.

9. Ge L, Yu J, Wang H, Luth D, Bai G, Wang K, Chen R. Increasing seed size and quality by manipulating BIG SEEDS1 in legume species. Proc Natl Acad Sci USA. 2016;113(44):12414-9.

10. Carter NP. Methods and strategies for analyzing copy number variation using DNA microarrays. Nat Genet. 2007;39(7 Suppl):S16-21.

11. Ge L, Chen R. Negative gravitropic response of roots directs auxin flow to control root gravitropism. Plant Cell Environ. 2019;42(8):2372-83.

12. Ye K, Schulz MH, Long Q, Apweiler R, Ning Z. Pindel: a pattern growth approach to detect break points of large deletions and medium sized insertions from paired-end short reads. Bioinformatics. 2009:25(21):2865-71.

13. Chen K, Wallis JW, McLellan MD, Larson DE, Kalicki JM, Pohl CS, McGrath SD, Wendl MC, Zhang Q, Locke DP, et al. BreakDancer: an algorithm for high-resolution mapping of genomic structural variation. Nat Methods. 2009;6(9):677-81.

14. Fan X, Abbott TE, Larson D, Chen K. BreakDancer: identification of genomic structural variation from paired-end read mapping. Curr Protoc Bioinformatics. 2014;45:15.

15. Sun L, GeY, Bancroft AC, Cheng X, Wen J. FNBtools: a software to identify homozygous lesions in deletion mutant populations. Front Plant Sci. 2018;9:976

16. Klein H, Xiao Y, Conklin PA, Govindarajulu R, Kelly JA, Scanlon MJ, Whipple CJ, Bartlett M. Bulked-segregant analysis coupled to whole genome sequencing (BSA-Seq) for rapid gene cloning in maize. G3 (Bethesda). 2018;8(11):3583-92.

17. Liang T, Chi W, Huang L, Qu M, Zhang S, Chen ZQ, Chen ZJ, Tian D, Gui Y, Chen X, et al. Bulked segregant analysis coupled with whole-genome sequencing (BSA-Seq) mapping identifies a novel pi21 haplotype conferring basal resistance to rice blast disease. Int J Mol Sci. 2020;21:6.

18. McKenna A, Hanna M, Banks E, Sivachenko A, Cibulskis K, Kernytsky A Garimella K, Altshuler D, Gabriel S, Daly M, et al. The Genome Analysis Toolkit: a MapReduce framework for analyzing next-generation DNA sequencing data. Genome Res. 2010;20(9):1297-303.

19. DePristo MA, Banks E, Poplin R, Garimella KV, Maguire JR, Hartl C, Philippakis AA, del Angel G, Rivas MA, Hanna M, et al. A framework for variation discovery and genotyping using next-generation DNA sequencing data. Nat Genet. 2011:43(5):491-8. 
20. Van der Auwera GA, Carneiro MO, Hartl C, Poplin R, Del Angel G, LevyMoonshine A, Jordan T, Shakir K, Roazen D, Thibault J, et al. From FastQ data to high confidence variant calls: the Genome Analysis Toolkit best practices pipeline. Curr Protoc Bioinform. 2013;43:11.

21. Poplin R, Ruano-Rubio V, DePristo MA, Fennell TJ, Carneiro MO, Van der Auwera GA, Kling DE, Gauthier LD, Levy-Moonshine A, Roazen D, et al. Scaling accurate genetic variant discovery to tens of thousands of samples. bioRxiv. 2018. https://doi.org/10.1101/201178.

22. Pecrix Y, Staton SE, Sallet E, Lelandais-Briere C, Moreau S, Carrere S, Blein T, Jardinaud MF, Latrasse D, Zouine M, et al. Whole-genome landscape of Medicago truncatula symbiotic genes. Nat Plants. 2018;4(12):1017-25.

23. Robinson JT, Thorvaldsdottir H, Winckler W, Guttman M, Lander ES, Getz G, Mesirov JP. Integrative genomics viewer. Nat Biotechnol. 2011;29(1):24-6.

24. Berrabah F, Bourcy M, Eschstruth A, Cayrel A, Guefrachi I, Mergaert P, Wen J, Jean V, Mysore KS, Gourion B, et al. A nonRD receptor-like kinase prevents nodule early senescence and defense-like reactions during symbiosis. New Phytol. 2014;203(4):1305-14.

25. Doll NM, Bovio S, Gaiti A, Marsollier AC, Chamot S, Moussu S, Widiez T, Ingram $\mathrm{G}$. The endosperm-derived embryo sheath is an anti-adhesive structure that facilitates cotyledon emergence during germination in arabidopsis. Curr Biol. 2020;30(5):909-15.

26. Chen G, Bi YR, Li N. EGY1 encodes a membrane-associated and ATPindependent metalloprotease that is required for chloroplast development. Plant J. 2005;41(3):364-75.
27. Chen Y, Wang X, Lu S, Wang H, Li S, Chen R. An array-based comparative genomic hybridization platform for efficient detection of copy number variations in fast neutron-induced Medicago truncatula Mutants. J Vis Exp. 2017;129:1

28. Sims D, Sudbery I, llott NE, Heger A, Ponting CP. Sequencing depth and coverage: key considerations in genomic analyses. Nat Rev Genet. 2014;15(2):121-32.

29. Gemenet DC, Lindqvist-Kreuze $H$, De Boeck B, da Silva PG, Mollinari M, Zeng ZB, Craig Yencho G, Campos H. Sequencing depth and genotype quality: accuracy and breeding operation considerations for genomic selection applications in autopolyploid crops. Theor Appl Genet. 2020;133(12):3345-63.

30. Liu CW, Breakspear A, Stacey N, Findlay K, Nakashima J, Ramakrishnan K, Liu M, Xie F, Endre G, de Carvalho-Niebel F, et al. A protein complex required for polar growth of rhizobial infection threads. Nat Commun. 2019;10(1):2848.

31. Li H, Handsaker B, Wysoker A, Fennell T, Ruan J, Homer N, Marth G, Abecasis G, Durbin R. Genome Project Data Processing S: The Sequence Alignment/Map format and SAMtools. Bioinformatics. 2009;25(16):2078-9.

\section{Publisher's Note}

Springer Nature remains neutral with regard to jurisdictional claims in published maps and institutional affiliations.
Ready to submit your research? Choose BMC and benefit from:

- fast, convenient online submission

- thorough peer review by experienced researchers in your field

- rapid publication on acceptance

- support for research data, including large and complex data types

- gold Open Access which fosters wider collaboration and increased citations

- maximum visibility for your research: over $100 \mathrm{M}$ website views per year

At BMC, research is always in progress.

Learn more biomedcentral.com/submissions 